\title{
Exploratory Approach to the Computational Modeling of Narrative Ability for Artificial Intelligence
}

\author{
Taisuke Akimoto
}

\begin{abstract}
Narrative ability is an essential element of human intelligence from the perspectives of both psychology and artificial intelligence (AI). It includes many intellectual functions: narrative generation, narrative understanding or interpretation, narrative-mediated communication, and the manipulation of narrativity-based knowledge. The computational modeling of narrative ability is a critical problem in the development of human-like AI agents and user-friendly intelligent information systems. However, implementing this model involves many difficult challenges owing to the structural and phenomenal complexity of narratives. The broader purpose of this study is to develop a computational model of narrative ability from an AI perspective. In this study, we explore the formulation of a conceptual framework of computational narrative ability including a narrativity-based knowledge model and operational modules for this knowledge model.
\end{abstract}

Index Terms-Artificial intelligence, computational narrative ability, narrative agent, story-form knowledge.

\section{INTRODUCTION}

In this paper, we discuss the computational modeling of narrative ability from the perspective of artificial intelligence (AI). A narrative is a common mode of organically representing events in the real or fictional world. Narrative ability includes many intellectual functions: narrative generation, narrative understanding, narrative-mediated communication, storage of narrativity-based knowledge, and retrieval and use of that knowledge.

The importance of narrative ability or narrativity in the human mind and AI has been discussed from the psychological as well as AI perspectives [1]-[4]. From a psychological perspective, Bruner [1] explains that the human experience and cognition of the real world is based on narrativity and is formed as narratives. Schank and his colleagues proposed several AI theories of narrativity-based knowledge models including script, goal-plan, theme, and story [5]. They also formulated the concept of case-based reasoning [6] as a model for solving a new problem by using a previous similar case and its solution. Mateas and Sengers [3], [4] introduced the term "narrative intelligence" to describe a multiple research perspective on the relationship between narrative and AI. In studies on cognitive architectures, several researchers have been involved in the modeling of episodic memory [7]. Soar cognitive architecture [8], for example, has episodic memory as one of the knowledge elements [9], [10].

Manuscript received September 13, 2016; revised December 20, 2016.

T. Akimoto is with the Graduate School of Informatics and Engineering, the University of Electro-Communications, Tokyo, Japan (e-mail: t8akimo@yahoo.co.jp).
Furthermore, the knowledge representation of episodic memory is discussed in terms of narrative or story [11]-[13].

The computational modeling of narrative creativity, including its implementation, has long been a challenging problem in the field of AI. Many researchers have studied this problem from various perspectives, thoughts, and methodologies [14]-[18]. The theoretical background of these studies includes cognitive science on narrative, such as a story grammar [19], and narratology, such as Propp's morphology of the folktale [20] and Genette's narrative discourse theory [21].

Based on the above background, this study explores the computational modeling of narrative ability from the AI perspective. Although many challenging problems exist in the computational modeling of narrative ability, this study particularly emphasizes three aspects of narrative ability-knowledge, communicative, and generative aspects. These aspects will be crucial elements for implementing human-like AI agents and user-friendly intelligent information systems for the following reasons.

As described previously, the knowledge aspect of narratives has long been discussed by AI researchers. At the same time, a narrative is a universal mode for communicating knowledge or information. This will continue to be true in the context of human-computer communications. However, few studies describe the computational modeling of narrative-mediated communication that includes dialogue with humans. In addition, the generative ability or creativity of narratives is necessary both for generative processes of internally constructing narrativity-based knowledge and expressing narratives to others. Although many studies on the computational modeling of narrative creativity have been conducted as previously cited, these studies have been mainly focused on literary creativity or entertainment. We attempt to expand this accumulated knowledge to come up with a more general model for computational narrative ability as a basis for intelligence.

In this paper, we discuss the above problems for formulating a conceptual framework of computational narrative ability. We also show an architectural design of a narrative agent to perform prototyping of a part of computational narrative ability.

The rest of the paper is organized as follows. Section II discusses several potential approaches to computational narrative ability as a basis for general intelligence. Sections III and IV formulate the concept of a "story-form knowledge" as the knowledge aspect of narratives. Section V provides a framework of operational modules for the story-form knowledge as a basis for the various intellectual functions of computational narrative ability. Section VI shows the design 
of a narrative agent involving part of computational narrative ability. Section VII concludes this paper.

\section{POTENTIAL APPROACHES TO COMPUTATIONAL NARRATIVE ABILITY}

In this section, we consider several potential approaches to computational narrative ability from a long-term perspective.

\section{A. Knowledge Sharing}

Narrative is a universal and effective mode of human communication, and hence, computational narrative ability is an essential element of human-agent communication. In particular, the ability of composing narratives through editing the internal knowledge of an agent will be a key element in providing rich and valuable information to humans. Conversely, the ability of narrative understanding or interpretation is also necessary for gaining knowledge from human narratives.

\section{B. Cooperative Problem Solving}

Narrative generation is similar to problem solving. Planning a series of actions for solving a problem is a type of narrative or story generation. Actually, AI techniques of problem solving such as goal-based planning and case-based reasoning are among the major approaches to computational narrative generation [14], [15], [22], [23]. In the context of problem solving, computational narrative ability will be a basis for creating a narrative as a solution, proposing it to humans, and improving it through interaction with humans.

\section{Cooperative Design}

In a manner similar to that of cooperative problem solving, computational narrative ability will also be applied to the process of designing a complex artifact such as a city environment, software program, business plan, and service. For example, considering a quality user experience story is a crucial task for creating a better product [24]. This type of narrative is composed by harmonizing various values from multiple perspectives. An agent with high narrative ability will be able to support this type of narrative creation in design processes.

\section{Constructing Identity}

From the psychological perspective, narrative ability or narrativity is assumed an essential nature of the human mind. Experiences in the real world are integrated into a consistent narrative of one's life in the human mind and the identity is constructed by positioning oneself in this life story. However, this kind of narrativity is absent from the mind of current AI agents, as pointed out by Sengers [25]. Computational narrative ability is necessary for composing one's life story or episodic memory as the foundation of an agent's identity. Furthermore, the ability to recite past experiences to humans in the form of a narrative is also important.

\section{E. Learning}

Humans learn diverse knowledge from narratives and form their common-sense knowledge, which includes linguistic knowledge, ontological knowledge, social convention, causality, emotion, and ethics. Although many challenges of learning from a huge amount of text data have been addressed in the area of machine learning [26], [27], the computational modeling of the knowledge aspect of narratives [2], [5], [28] will be a key problem related to the learning ability from narratives.

\section{NARRATIVE AS KNOWLEDGE}

A narrative generally refers to an output of narrating one or more events. Its expression media include natural language (utterance or text) and still or moving images. At the same time, narrative is also described as a comprehensive form of knowledge or memory. These two aspects, expressed and internal narratives, are illustrated in Fig. 1. This section discusses the knowledge aspect of narratives from the following two perspectives:

1) A. We discuss the difference between the expressed and internal narratives and introduce the term "story-form knowledge" for distinguishing them.

2) B. We provide a hypothetical classification of the story-form knowledge.

\section{A. Story-Form Knowledge}

In considering the difference between internal and expressed narratives, we refer to the narratological terminologies of dividing the content (or what to tell) and expression (or how to tell) planes of a narrative, i.e., a "story" (also known as a "fabula") and a "discourse" (also known as a "sujet") [21], [29]. These terms are also used in recent studies on the computational modeling of narrative [30], [31], especially in narrative generation systems [18], [32], [33].

A story (content plane) involves chronologically ordered events and its background world including entities such as humans, items, and places. In the discourse plane, one story can be expressed in various manners in terms of the temporal order (e.g., flashback and flash-forward), speed or tempo (e.g., summary and stretch), perspective or point-of-view, and so on [21].

From the perspective of knowledge representation, we assume that an internal narrative corresponds to the source of expressed narratives. It is similar to the story rather than the discourse. Therefore, we introduce the term "story-form knowledge" for referring to the knowledge aspect of narratives. However, an expressed narrative corresponds to a discourse; the generation of an expressed narrative for communicating story-form knowledge to others is formalized as a discourse generation.

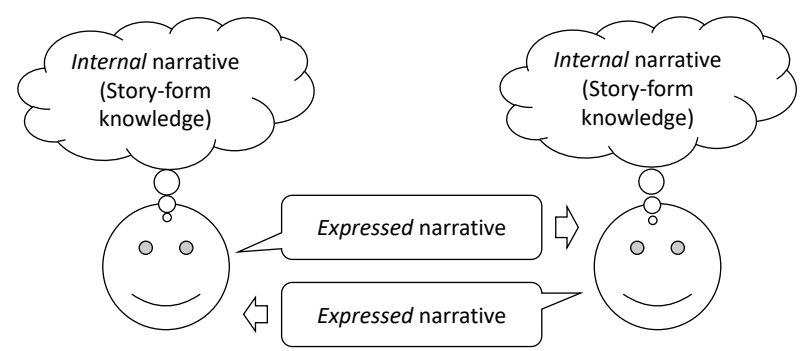

Fig. 1. Internal and expressed narratives.

\section{B. Classification of Story-Form Knowledge}

We assume that the concept of story-form knowledge can 
be applied to several knowledge types. The previous studies on narrativity-based knowledge representation have mainly dealt in terms of an episodic memory or experience, procedural memory or script, and more comprehensive story [2], [5], [11], [12], [13], [28]. However, we focus on the manner of learning or constructing story-form knowledge and propose three knowledge types: experience-based, narrative-mediated, and internally generated stories.

\section{1) Experience-based stories}

An experience-based story refers to a story constructed based on one's own experiences in the real or virtual world. This is similar to the concept of episodic memory [7]. The past of an agent is formed by these stories.

\section{2) Narrative-mediated stories}

A narrative-mediated story refers to a story acquired from a narrative (e.g., novel, news report, conversation) as a human learns many stories from narratives beyond one's own experiences. This type of stories can be further classified into two types: factual and fictional stories.

\section{a) Factual stories}

Factual stories are collected from narratives about events in the real world. For example, the stories narrated in news reports and historical texts can be basically viewed as facts. Factual stories also include experiences of others told in their narratives.

\section{b) Fictional stories}

Fictional stories, including realistic and unrealistic stories, are collected from fictional narratives including novels, films, dramas, and computer games.

Note that the border of fact and fiction involves ambiguity. Factual narratives (e.g., news reports) may contain fictional stories including lies and errors. Similarly, fictional narratives (e.g., novels) may contain factual stories. Hence, we assume that the distinction between fact and fiction is basically left to one's interpretation. This hypothesis is also applied to the distinction between realism and unrealism.

\section{3) Internally generated stories}

An internally generated story refers to a story constructed inside the mind of an agent. This type of story includes future, imaginative, and generalized stories.

\section{a) Future stories}

A future story is similar to a plan for directing one's actions in the short- to long-term future. In many cases, future stories are constructed based on one's goals, desires, or dreams. The ability to produce future stories is a sign of intelligence relevant to problem solving by using techniques such as goal-based planning and case-based reasoning. The techniques of planning and case-based reasoning are also applied to narrative generation systems [14], [15], [22], [23].

\section{b) Imaginative stories}

An imaginative story refers to a fictional story imagined inside the mind of an agent. We assume that imaginative stories are mainly composed in creating fictional works of literature or other genres of narrative. In such cases, a created work corresponds to an expression of an imaginative story. In addition, daydreams are also included in this category (refer to [34] for an AI model of daydreaming).

\section{c) Generalized stories}

In general terms, a story basically has concreteness and specialty with specific events, characters, times, places, etc. However, schematic abstract knowledge models for constructing and/or understanding stories, including script [5], procedural knowledge [13], and story grammar [19], will also be assumed as types of story-form knowledge. These types of knowledge will be constructed through an abstraction or generalization of concrete stories including experience-based and narrative-mediated stories.

\section{REPRESENTATION OF STORY-FORM KNOWLEDGE}

Next, we consider a structural model for representing the story-form knowledge. For this purpose, we apply a computational model of narrative structure representation as proposed in [35]. Although this model has been designed with a focus on the expressed narrative, we assume that a huge commonality exists between the internal and expressed narratives in their structures.

\section{A. Hierarchical Graph Model for Narrative Structure}

A hierarchical graph model for narrative structure representation is proposed in [35], in which various types of narrative units are organized hierarchically, with network-like linkages. It is based on the concept of object-oriented modeling. In this model, each of the story and discourse (in terms of narratology) aspects are further divided into two dimensions (see Fig. 2). In particular, a story consists of a StoryWorld and a Story (in a narrow sense) and a discourse consists of a Discourse (in a narrow sense) and an Expression. The four dimensions are defined below.

1) StoryWorld: The background world structure that contains information about entities (e.g., characters, objects, and places).

2) Story: The structure of chronologically organized events.

3) Discourse: The structure of how a Story is organized into a surface expression.

4) Expression: The surface expression using natural language, still or moving images, sounds, or other forms of media.

A Discourse is similar to the intermediate structure between content and expression. This type of intermediate structure is generally used in narrative generation systems as well as in natural language generation systems [36].

The hierarchical graph model is applied to each structural dimension of StoryWorld, Story, and Discourse. A hierarchical graph consists of three types of structural components: a semantic element (SE), a Group, and a Link.

- The $S E$ contains semantic information for the basic unit in each structural dimension of a narrative.

- The Group integrates any number of nodes (SES and/or Groups) into a broader unit. This function is similar to the linguistic abstraction or categorization of the member nodes. A Group itself has semantic information in the form of a comprehensive meaning that binds or abstracts the member nodes.

- The Link connects any two nodes with a relation or a set of connective attributes. 


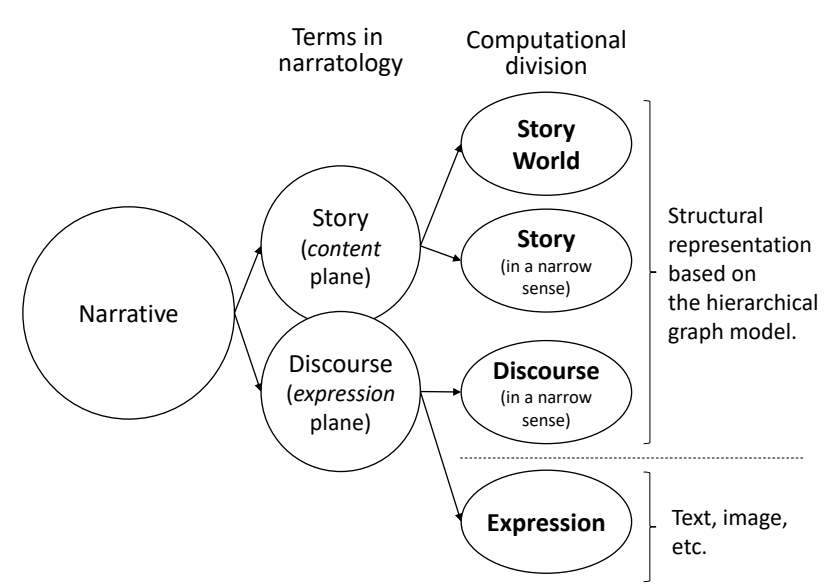

Fig. 2. Four dimensions of a narrative.

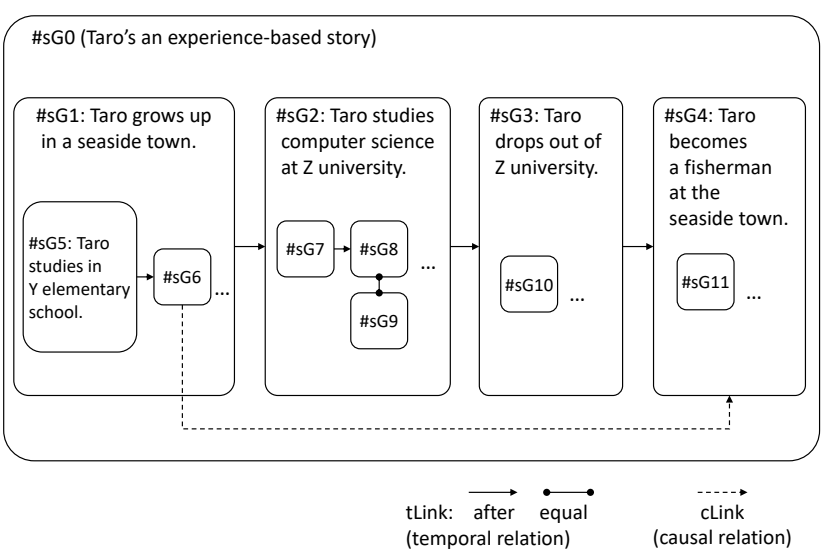

Fig. 3. An example Story structure for an experience-based story of an agent "Taro".

Although the components are commonly used in any structural dimension, the content of each type is different in every dimension. The subclasses of SE, Group, and Link for each dimension are defined in Table I.

\section{B. Applying the Hierarchical Graph Model to Story-Form Knowledge}

By using the above model, a unit of story-form knowledge, i.e., a story, can be represented as a pair of a StoryWorld and a Story. We use the term "k-story" for referring to this knowledge unit, in order to avoid confusion between a Story in the hierarchical graph model and a story as a knowledge unit. A knowledge base of story-form knowledge, referred to as "k-story base," stores a number of k-stories including experience-based, narrative-mediated, and internally generated stories.

Fig. 3 illustrates an example Story structure for a k-story. This k-story assumes an experience-based story of an agent "Taro." The attribute information of entities in this Story (e.g., "Taro," "Seaside town," "Y elementary school," and "Z university") are represented in the corresponding StoryWorld (we omit the explanation of this StoryWorld for simplicity).

The Story structure represents a temporal organization of events based on sGroups and tLinks. tLinks define the chronological order of events and sGroups form a temporal hierarchical segmentation of events from the summarized level to the detailed level.

Causality among events is also an important aspect for a k-story. This is defined by the cLink. In this example (Fig. 3), two sGroups "sG6" and "sG4" are related with a cLink. This structure means that "\#sG6"- an experience of "Taro" such as "Taro falls into the sea. An older fisherman rescues Taro. Taro expresses his admiration for the fisherman."-is positioned as the reason by which "Taro" becomes a fisherman ("sG4").

TABLE I: SUBCLASSES OF THE STRUCTURAL COMPONENTS OF THE HIERARCHICAL GRAPH MODEL

\begin{tabular}{|c|c|c|}
\hline \multirow{3}{*}{ 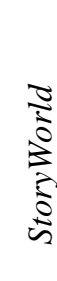 } & Entity (SE) & $\begin{array}{l}\text { An individual existence; for example, human, } \\
\text { object, and place. }\end{array}$ \\
\hline & wGroup & $\begin{array}{l}\text { An aggregation of Entities/wGroups, such } \\
\text { as a human group, a spatial unit, and } \\
\text { functional kinship. }\end{array}$ \\
\hline & wLink & $\begin{array}{l}\text { s; for example, } \\
\text { tial relations. }\end{array}$ \\
\hline \multirow[b]{3}{*}{$\sum^{5}$} & Event (SE) & An a \\
\hline & sGroup & $\begin{array}{l}\text { egation of Events/sGroups, such as } \\
\text { or a broader unit. }\end{array}$ \\
\hline & $\begin{array}{l}\text { tLink and } \\
\text { cLink }\end{array}$ & $\begin{array}{l}\text { A think is a relative temporal relation } \\
\text { between two nodes for chronologically } \\
\text { organizing a Story; for example, "before" } \\
\text { and "equal." } \\
\text { A cLink is a causal relation between two } \\
\text { nodes. }\end{array}$ \\
\hline \multirow[b]{3}{*}{ 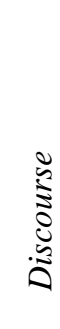 } & $D L$ & A minimum serment of a Diccourse \\
\hline & $d G r$ & $\begin{array}{l}\text { A broader unit of DUs/dGroups as a } \\
\text { semantic or formal segment of a Discourse. }\end{array}$ \\
\hline & $d \operatorname{Link}$ & $\begin{array}{l}\text { A pathway between two nodes with } \\
\text { connective logic. That is, the threads of a } \\
\text { narrative are composed using dLinks, } \\
\text { defining the order in which the DUs are } \\
\text { expressed. }\end{array}$ \\
\hline
\end{tabular}

However, the hierarchical graph model has several limitations. First, it lacks theories for representing one's subjective value or meaning of events and entities, such as the polarity and degree of importance of an event. This type of information will be positioned as an accompaniment to a node (SE or Group) in the hierarchical graph structure. Second, the hierarchical graph model cannot be applied to the representation of a generalized story (see Section III-B-3-c). Another representation model is required for generalized stories.

\section{OPERATIONAL MODULES FOR THE STORY-FORM KNOWLEDGE}

In this section, we consider the operational modules relevant to the story-form knowledge for performing various intellectual tasks. Totally, we provide six basic operations: story generation, discourse generation, organization, retrieval, generalization, and narrative interpretation (Fig. 4). The following subsections explain the role of each operation.

\section{A. Story Generation}

The function of the story generation module is to construct k-stories, especially experience-based, future, and imaginative stories in the aforementioned classification (Section III-B). An ability to generate future and imaginative stories is especially relevant to the creative aspect of intelligence. For example, it will be used in considering a 
better future plan and plotting a unique story for an entertainment narrative.

We assume that the construction of experience-based stories involves subjective cognitive processes to integrate sensory information and create a consistent story. This process includes segmenting events from continuous sensory data [11] and relating among events. Therefore, the construction of experience-based stories is positioned as a function of the story generation module.

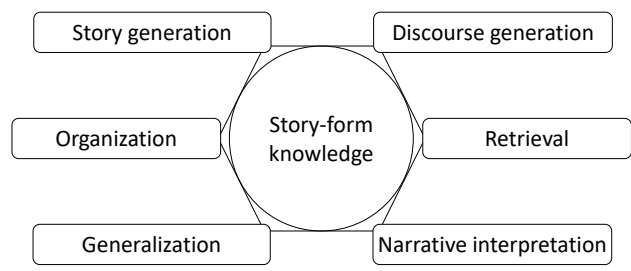

Fig. 4. Operational modules for the story-form knowledge.

\section{B. Discourse Generation}

The discourse generation module produces a narrative expression by using the k-story base with a communicative or creative goal. This module consists mainly of the following three generative processes: 1) retrieving one or more k-stories from the k-story base with the retrieval module, 2) composing a Discourse structure from the retrieved k-stories, and 3) generating a natural language text (an Expression).

In several studies on computational narrative generation [18], [32], [33], narrative discourse generation has been designed based on Genette's narratology [21] that systematically categorizes various classes of discourse structures through the analysis of a novel. These computational models commonly formalize the narrative discourse generation process with a set of transformational techniques from a story structure to a discourse structure. These computational methods can be applied to this discourse generation module, particularly to step 2 in the aforementioned procedure.

\section{Organization}

This module organizes a large amount of k-stories in the $\mathrm{k}$-story base for increasing the accessibility for other modules. The types of knowledge organization may include network-like linkage among k-stories and hierarchical grouping of similar k-stories. This module also includes the function of forgetting k-stories.

\section{Retrieval}

The retrieval module searches for k-stories from the k-story base on request from other modules. For example, when an agent talks about a childhood episode, the discourse generation module will request a corresponding part of experience-based stories to the retrieval module.

\section{E. Generalization}

The generalization module constructs a generalized story from one or more k-stories in the k-story base. This module will be one of the basic elements for learning from narratives or experiences.

\section{F. Narrative Interpretation}

This module interprets an expressed narrative and composes a k-story as the result. This module plays a key role in constructing narrative-mediated stories.

\section{ARChitecture Design of a NARRAtive AgENT: PlaN FOR A PROTOTYPE DEVELOPMENT}

We plan to prototype a narrative agent as an implementation of a part of the computational narrative ability.

\section{A. Outline}

Fig. 5 shows an architecture design of the narrative agent prototype. This agent has the ability to tell k-stories in a k-story base through text-based dialogues with a human. The k-story base stores only experience-based and future stories, including one's goals, desires, and dreams. A human will understand or feel the agent's background, individuality, and personality through a dialogue with the agent.

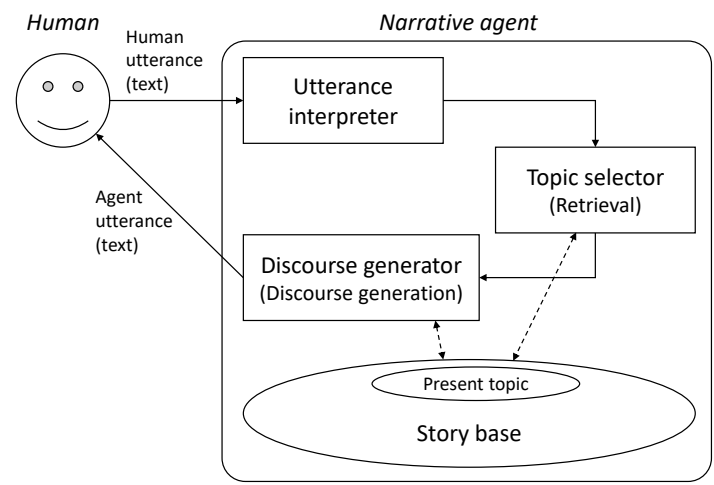

Fig. 5. Architecture design of a narrative agent.

The process flow of the narrative agent is summarized below:

Step 1. An utterance interpreter receives a human utterance (text) and analyzes it for determining queries to be used in the k-story base retrieval in step 2 .

Step 2. A topic selector retrieves a node of a k-story (an sGroup or Event in the Story structure) from the k-story base according to the queries determined in step 1 . The retrieved node is set as the present topic to be narrated in the next agent utterance.

Step 3. A discourse generator composes a Discourse structure by using the present topic and then generates natural language sentences (Expression) from the Discourse.

The generated sentences are presented to the human and the above processes are repeated until the human exits the dialog.

\section{B. Main Problems}

This prototype version implements the following three key functionalities of the computational narrative ability.

\section{1) Modeling a knowledge representation for the} story-form knowledge

We will implement a representation model of the story-form knowledge based on the consideration in Section IV. By using this model, a number of hand-created k-stories including experience-based and future stories are installed in the k-story base.

\section{2) Implementing "retrieval" for the topic selector}

A key function of the topic selector is to choose an 
appropriate topic for an agent utterance according to a human utterance. For example, when the agent is asked by a human to talk about a childhood episode, the topic selector should retrieve a part of a k-story relevant to one's childhood experiences.

\section{3) Implementing "discourse generation" for the discourse generator}

In the simplest case, the discourse generator produces sentences in which the events of a selected topic (retrieved node) are plainly recounted in a chronological order. However, a significant role of the discourse generator is to narrate k-stories by various methods. In particular, we plan to introduce the following discourse techniques by referring to Genette's narratology [21].

- Temporal transition: The agent occasionally changes the present topic from the one selected by the topic selector to another topic in mid-sentence, by relating the two topics in an associative manner.

- Hiding: The agent occasionally omits negative events (or uncomfortable events to talk about, such as failures and crimes) from the present topic.

- Summary and stretch: The agent controls the detail level of narrating a topic by using the hierarchical grouping structure of the Story.

- Commentary and description: The agent describes not only events, but also commentaries or descriptions on humans, places, or other entities. For example, the agent first explains the key persons involved in the present topic.

- First-person: In agent's narratives, the agent himself is designated as the first-person.

\section{CONCLUSION}

In this paper, we described the major problems encountered in the computational modeling of narrative ability from an AI perspective. We mainly discussed two aspects of narrative ability: the story-form knowledge that represents the knowledge aspect of narratives and the operational modules for the story-form knowledge that provide a basic framework for various intellectual functions of the computational narrative ability. We also described the prototype design of a narrative agent including part of the computational narrative ability.

Through these discussions, we provided the draft of a new research framework for the computational narrative ability. This framework is based on the reorganization of previous AI studies on narratives, especially narrativity-based knowledge models and computational narrative generation. Although many challenging problems were addressed in this study, narrative ability will be a crucial element for human-like AI agents. Our future work will involve prototyping a narrative agent and providing conceptual and architectural refinement to the computational narrative ability.

\section{REFERENCES}

[1] J. Bruner, "The narrative construction of reality," Critical Inquiry, vol. 18, no. 1, pp. 1-21, 1991.

[2] R. C. Schank, Tell Me a Story: Narrative and Intelligence, Chicago, IL: Northwestern University Press, 1990.
[3] M. Mateas and P. Sengers, "Narrative intelligence," Narrative Intelligence: Papers from the AAAI Fall Symposium, Technical Report FS-99-01, 1999.

[4] M. Mateas and P. Sengers, Narrative Intelligence, Amsterdam, the Netherlands: John Benjamins Publishing, 2003.

[5] R. C. Schank and R. P. Abelson, Scripts, Plans, Goals, and Understanding: An Inquiry into Human Knowledge Structures, Mahwah, NJ: Lawrence Erlbaum, 1977.

[6] C. K. Riesbeck and R. C. Schank, Inside Case-Based Reasoning, Mahwah, NJ: Lawrence Erlbaum, 1989.

[7] E. Tulving, Elements of Episodic Memory, Oxford, U.K.: Oxford University Press, 1983.

[8] J. E. Laird, The Soar Cognitive Architecture, Cambdidge, MA: The MIT Press, 2012.

[9] A. M. Nuxoll and J. E. Laird, "A cognitive model of episodic memory integrated with a general cognitive architecture," in Proc. International Conference on Cognitive Modeling, pp. 220-225, 2004

[10] A. M. Nuxoll and J. E. Laird, "Extending cognitive architecture with episodic memory," in Proc. the 22nd National Conference on Artificial Intelligence, pp. 1560-1565, 2007.

[11] T. S. Anderson, "From episodic memory to narrative in a cognitive architecture," in Proc. 6th Workshop on Computational Models of Narrative, pp. 2-11, 2015.

[12] N. Szilas, "Towards narrative-based knowledge representation in cognitive systems," in Proc. 6th Workshop on Computational Models of Narrative, pp. 133-141, 2015.

[13] C. León, "An architecture of narrative memory," Biologically Inspired Cognitive Architectures, vol. 16, pp. 19-33, 2016.

[14] J. R. Meehan, The Metanovel: Writing Stories by Computer, New York, NY: Garland Publishing, 1980.

[15] S. R. Turner, The Creative Process: A Computer Model of Storytelling and Creativity, Mahwah, NJ: Lawrence Erlbaum, 1994.

[16] S. Bringsjord and D. A. Ferrucci, Artificial Intelligence and Literary Creativity: Inside the Mind of BRUTUS, a Storytelling Machine, Mahwah, NJ: Lawrence Erlbaum, 1999.

[17] P. Gervás, "Computational approaches to storytelling and creativity," AI Magazine, vol. 30, no. 3, pp. 49-62, 2009.

[18] T. Ogata, "Computational and cognitive approaches to narratology from the perspective of narrative generation," in Computational and Cognitive Approaches to Narratology, T. Ogata \& T. Akimoto Eds. Hershey, PA: IGI Global, 2016, ch. 1, pp. 1-74.

[19] D. E. Rumelhart, "Notes on a schema for stories," in Representation and Understanding: Studies in Cognitive Science, D. G. Bobrow \& A. Collins Eds. Waltham, MA: Academic Press, 1975.

[20] V. Y. Propp, Morphology of the Folktale, Trans. L. Scott, Austin, TX: University of Texas Press, 1968. (Original work published 1928).

[21] G. Genette, Narrative Discourse: An Essay in Method, NY: Cornell University Press, 1980. (Original work published 1972)

[22] P. Gervás, B. Díaz-Agudo, F. Peinado, and R. Hervás, "Story plot generation based on CBR," Knowledge-Based Systems, vol. 18, no. 4-5, pp. 235-242, 2005.

[23] M. O. Riedl and R. M. Young, "Narrative planning: Balancing plot and character," Journal of Artificial Intelligence Research, vol. 39, pp. 217-267, 2010.

[24] W. Quesenbery and K. Brooks, Storytelling for User Experience: Crafting Stories for Better Design, New York, NY: Rosenfeld Media, 2010.

[25] P. Sengers, "Schizophrenia and narrative in artificial agents," in Narrative Intelligence, M. Mateas \& P. Sengers Eds. Amsterdam, the Netherlands: John Benjamins Publishing, 2003, ch. 16, pp. 259-278.

[26] H. Liu and P. Singh, "ConceptNet: A practical commonsense reasoning tool-kit," BT Technology Journal, vol. 22, no. 4, pp. 211-226, 2004.

[27] N. Chambers and D. Jurafsky, "A database of narrative schemas," in Proc. Seventh International Conference on Language Resources and Evaluation, pp. 1614-1618, 2010.

[28] R. C. Schank and R. P. Abelson, "Knowledge and memory: The real story," in Knowledge and Memory: The Real Story, R. S. Wyer, Jr. Ed. Hillsdale, NJ: Lawrence Erlbaum, 1995, ch. 1, pp. 1-85.

[29] G. Prince, A Dictionary of Narratology, Lincoln, NE: University of Nebraska Press, 2003.

[30] P. Gervás, B. Lönneker-Rodman, J. C. Meister, and F. Peinado, "Narrative models: Narratology meets artificial intelligence," in Proc. Satellite Workshop: Toward Computational Models of Literary Analysis, 5th International Conference on Language Resources and Evaluation, pp. 44-51, 2006.

[31] I. Mani, Computational Modeling of Narrative, San Rafael, CA: Morgan \& Claypool Publishers, 2013. 
[32] N. Montfort, "Curveship: An interactive fiction system for interactive narrating," in Proc. the NAACL HLT Workshop on Computational Approaches to Linguistic Creativity, pp. 55-62, 2009.

[33] T. Akimoto and T. Ogata, "A narratological approach for narrative discourse: Implementation and evaluation of the system based on Genette and Jauss," in Proc. the 34th Annual Conference of the Cognitive Science Society, pp. 1272-1277, 2012.

[34] E. T. Mueller, Daydreaming in Humans and Machines, Norwood, NJ: Ablex, 1990.

[35] T. Akimoto, "Toward a basic model of narrative structure representation for computer processing: Proposal of a hierarchical graph model," in Proc. the International Workshop on Language Sense on Computer in IJCAI2016, pp. 9-16, 2016.

[36] E. Reiter and R. Dale, Building Natural Language Generation Systems, Cambridge, U.K.: Cambridge University Press, 2000.

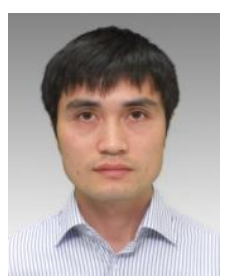

Taisuke Akimoto is a postdoctoral researcher in the Graduate School of Informatics and Engineering, the University of Electro-Communications, Tokyo, Japan. $\mathrm{He}$ received his $\mathrm{Ph} . \mathrm{D}$. from Iwate Prefectura University, Japan, in 2014. His research interests include artificial intelligence, informatics, and cognitive science. Especially, he interests automatic narrative generation system, computational modeling of narrative ability, and their applications. He is a member of the Japanese Society for Artificial Intelligence, Japanese Cognitive Science Society, and the Association for Natural Language Processing in Japan. 